Pacific Journal of Mathematics

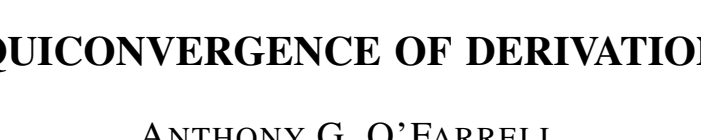




\title{
EQUICONVERGENCE OF DERIVATIONS
}

\author{
A. G. O'FARRELL
}

This paper is a study of bounded point derivations on the classical Banach algebras of analytic functions of a complex variable. The results are positive in character. The higher-order Gleason metrics $d^{p}$ of $R(X)$ are introduced and conditions are studied under which convergence takes place with respect to these metrics. In particular, if $R(X)$ admits a $p$ th-order bounded point derivation at a point $x \in \partial X$ and $\dot{X}$ satisfies a cone condition at $x$, then $d^{p}(y, x)$ tends to 0 as $y$ tends to $x$ along the midline of the cone. Similar results hold for the other classical function algebras. In the case of the algebra $H^{\infty}(U)$, for open $U \subset C$, the analogous results hold only for regular derivations (a regular $p$ th-order derivation maps $z^{p}$ to a nonzero complex number). The points of the maximal ideal space of $H^{\infty}(U)$ at which regular bounded point derivations exist are characterized in terms of analytic capacity, following Hallstrom.

1. Let $x$ be a point of the plane $C$ and $A$ be a class of functions analytic in a disc $D$ centered at $x$, each function having modulus bounded by 1 . Then, as is clear from Cauchy's integral formula, the family $\left\{f^{\prime} \mid f \in A\right\}$ is equicontinuous at $x$, and for every sequence $\left\{x_{n}\right\} \rightarrow x$, the sequence $\left\{f^{\prime}\left(x_{n}\right)\right\}$ converges to $f^{\prime}(x)$, uniformly on $A$, i.e., $\left\{f^{\prime}\left(x_{n}\right)\right\}$ is equiconvergent to $f^{\prime}(x)$. More generally, for any integer $p \geqq 1,\left\{f^{(p)}\left(x_{n}\right)\right\}$ is equiconvergent to $f^{(p)}(x)$.

Now, given a $C$-algebra $A$ of continuous functions on a compact set $X \subset C$ which are analytic on $\dot{X}^{\circ}$, it is often possible to find points on $\partial X$ at which nonzero point derivations exist on $A$. $A$ (first order) point derivation at $x \in X$ on $A$ is a linear functional $D: A \rightarrow C$ such that

$$
D(f g)=f(x) D g+g(x) D f,
$$

whenever $f, g \in A$. This notion generalizes that of derivative at a point. For points $y \in \stackrel{\circ}{X}$ all point derivations are of the form $f \rightarrow \alpha f^{\prime}(y)$ for some complex constant $\alpha$ (independent of $f$ ) provided $A$ contains the polynomials. Suppose $A$ contains the identity map $z$ and $D$ is a normalized point derivation at $x$ on $A$, i.e., $D z=1$. A natural question is:

Q1. When is there a sequence of points $x_{n} \in \dot{X}$, converging to $x$, such that the sequence $\left\{f^{\prime}\left(x_{n}\right)\right\}$ converges to Df for all $f \in A$ ?

A bounded point derivation is a point derivation that is continuous 
with respect to the uniform norm on $X$. If $A$ admits a bounded point derivation $D$ at a point $x$ we may ask:

Q2. Can we find $x_{n} \rightarrow x, x_{n} \in \dot{X}$, such that $f^{\prime}\left(x_{n}\right)$ is equiconvergent to Df on $A_{1}=A \cap\left\{f \mid\|f\|_{x} \leqq 1\right\}$ ?

We shall concern ourselves with Q2, which lends itself to treatment by Banach algebra techniques.

2. We treat first the case $A=R(X)$, the uniform closure on $X$ of $R_{0}(X)$, the class of rational functions with poles off $X . R(X)$ is a function algebra on $X[2, \mathrm{p} .2]$. The Gleason metric $d^{0}$ on $X$, with respect to $R(X)$, is defined by

$$
d^{0}(x, y)=\sup \left\{\mid f(x)-f(y)\left\|f \in R_{0}(X),\right\| f \|_{X} \leqq 1\right\},
$$

for $x, y \in X$. Here $\|f\|_{X}$ denotes the sup norm of $F$ on $X$. The properties of $X$ with respect to this metric have been thoroughly investigated. An account may be found in [2], [4]. If $x$ and $y$ belong to the same component of $\dot{X}$, then $d^{0}(x, y)<2$. If $x$ is a peak point for $R(X)$, then $d^{0}(x, y)=2$ whenever $y \neq x$. This prompted the definition of Gleason part. A part $P$ of the algebra $R(X)$ is a subset of $X$ which forms an equivalence class under the relation $x \sim y\left\langle\Rightarrow d^{0}(x, y)<2\right.$. The structure of parts can be very complicated. Davie has shown that $P$ may be disconnected, and the Swiss cheese example shows that $P$ may have no interior (cf. [4]). However, a nontrivial part (a part which does not just consist of one peak point) has full area density at each of its points, and in fact Browder $\left[2\right.$, p. 177] has shown that every Gleason ball $\left\{x \in X \mid d^{0}(x, a)<\varepsilon\right\}(\varepsilon>0)$ about a nonpeak point $a$ has full area density at $a$.

In particular, $a$ is not isolated in the part metric $d^{0}$, and there is a sequence of points $x_{n} \in P \backslash\{a\}$ which converges to a simultaneously in the Euclidean and Gleason metrics. In plain language, as $n \rightarrow+\infty$, $\left|x_{n}-a\right| \rightarrow 0$, and $\left\{f\left(x_{n}\right)\right\}$ is equiconvergent to $f(a)$ for $f \in R_{0}(X) \cap$ $\left\{f \mid\|f\|_{X} \leqq 1\right\}=R_{0}(X, 1)$.

For $p \geqq 1$ we define the $p$ th order Gleason metric on $X$ by

$$
d^{p}(x, y)=\sup \left\{\left|f^{(p)}(x)-f^{(p)}(y)\right| \mid f \in R_{0}(X, 1)\right\},
$$

for $x, y \in X$.

The first thing to note is that $d^{p}(x, y)$ may be $+\infty$, so we are using the word "metric" a little loosely. An ordinary metric may be obtained from $d^{p}$ by composing it with the arctangent function, but we would rather not do this. We extend $d^{p}$ to $\boldsymbol{C} \times \boldsymbol{C}$ by writing 
$d^{p}(x, y)=d^{p}(y, x)=+\infty$ whenever one of the elements $x, y$ fails to be in $X$.

For $p \geqq 0$ we say that a (normalized) $p$ th order bounded point derivation on $R(X)$ exists at a point $x \in X$ if and only if the functional $f \rightarrow f^{(p)}(x)$ on $R_{0}(X)$ extends to a continuous linear functional $D_{x}^{p}$ on $R(X)$, i.e., if and only if

$$
s^{p}(x)=\sup \left\{\left|f^{(p)}(x)\right| \mid f \in R_{0}(X, 1)\right\}=\left\|D_{x}^{p}\right\|
$$

is finite. Suppose this happens, and $x_{n}$ is a sequence of points of $\dot{X}$ tending to $x$ (in Euclidean norm). Then to say that $f^{(p)}\left(x_{n}\right) \rightarrow D_{x}^{p} f$ equiconvergently on $R(X, 1)$ is the same thing as saying that $d^{p}\left(x_{n}, x\right) \rightarrow 0$.

Notice that the two definitions so far available for a normalized first order bounded point derivation on $R(X)$ agree.

For purposes of computation it is usually easier to work with the function $d_{0}^{p}$, defined by

$$
d_{0}^{p}(x, y)=\sup \left\{\left|f^{(p)}(y)\right| \mid f \in R_{0}(X, 1) \text { and } f(x)=f^{\prime}(x)=\cdots=f^{(p)}(x)=0\right\} \text {. }
$$

3. The elementary properties of the functions $d^{p}, s^{p}, d_{0}^{p}$ are summarized in the following theorem. Here, as usual, $p$ is a nonnegative integer.

THEOREM 1. Let $x, y \in C$. Then

(1) $\left|s^{p}(x)-s^{p}(y)\right| \leqq d^{p}(x, y) \leqq s^{p}(x)+s^{p}(y)$;

(2) $\quad d^{p}(x, y) \geqq(p+1) !|x-y| /(\operatorname{diam} X)^{p+1}$;

(3) for $x \in X^{\circ}$,

$$
s^{p+1}(x)=\lim _{y \rightarrow x} \frac{d^{p}(x, y)}{|x-y|}
$$

(4) for each compact subset $K$ of a component of $\dot{X}$ there is a constant $L>0$ such that

$$
d^{p}(x, y) \leqq L|x-y|,
$$

for $x, y \in K$, so $d^{p}$ is continuous on $\dot{X}$;

(5) $s^{p}$ is continuous on $\stackrel{\dot{X}}{\text {; }}$

(6) $\quad d_{0}^{p}(x, y) \leqq d^{p}(x, y) \leqq\{1+\exp (\operatorname{diam} X)\}\left\{\sup _{0 \leqq \nu \leqq p} s^{\nu}(x)\right\} d_{0}^{p}(x, y)$;

(7) if $X_{n}$ is a decreasing sequence of compact sets, each containing $X$ in its interior, whose intersection is $X$, then $s_{n}^{p} \uparrow s^{p}$ and $d_{n}^{p} \uparrow d^{p}$, where $s_{n}^{p}$ and $d_{n}^{p}$ are respectively, the $s^{p}$-function and the $d^{p}$-function associated with $X_{n}$; 
(8) $s^{p}$ and $d^{p}$ are lower semi-continuous;

(9) if $\left|x_{n}-x\right| \rightarrow 0$ and $\left\{s^{p}\left(x_{n}\right)\right\}$ is a bounded sequence, then $s^{p}(x)$ is finite;

(10) if $s^{p}(w)<+\infty$ for some $w \neq x$, then $s_{p}(x)=+\infty$ if and only if $d^{p}(x, y)=+\infty$ for every $y \neq x$;

(11) $x$ is an interior point of $X$ if and only if

$$
\sup _{n \geqq 1}\left[\frac{1}{n} \log \frac{s_{n}(x)}{n !}\right]<+\infty \text {. }
$$

Proof.

(1) is clear.

(2): Take $f(z)=(z-y)^{p+1} /(\operatorname{diam} X)^{p+1}$. Then $f \in R_{0}(X, 1)$, so

$$
d^{p}(x, y) \geqq\left|f^{(p)}(x)-f^{(p)}(y)\right| \cdot
$$

(3) requires a lengthy but straightforward argument, using the Cauchy integral formula.

(4) follows from (3), using compactness.

(5) follows from (1) and (4).

(6): For the second inequality, let $f \in R_{0}(X, 1)$, and form

$$
g(z)=f(z)-\sum_{\nu=0}^{p} \frac{f^{(\nu)}(x)}{\nu !}(z-x)^{\nu} .
$$

Then $g(x)=g^{\prime}(x)=\cdots=g^{(p)}(x)=0$, and

$$
\begin{aligned}
\|g\|_{X} & \leqq 1+\sum_{\nu=0}^{p} \frac{s^{\nu}(x)}{\nu !}(\operatorname{diam} X)^{\nu} \\
& \leqq\left\{1+\sum_{\nu=0}^{p} \frac{(\operatorname{diam} X)^{\nu}}{\nu !}\right\}\left\{\sup _{0 \leqq \nu \leqq p} s^{\nu}(x)\right\} \\
& \leqq\{1+\exp (\operatorname{diam} X)\}\left\{\sup _{0 \leqq \nu \leqq p} s^{\nu}(x)\right\} .
\end{aligned}
$$

(7) follows from the fact that each $f \in R_{0}(X, 1)$ belongs to every $R\left(X_{n}\right)$ from some point on.

(8): By (4), (5), and (7), $s^{p}$ and $d^{p}$ are increasing limits of continuous functions.

(9): Take $X_{m} \downarrow X$ as in (7). For each $m, x \in \dot{X}_{m}$, so by (5),

$$
\begin{aligned}
s_{m}^{p}(x) & \leqq \sup _{n \geqq 1} s_{m}^{p}\left(x_{n}\right) \\
& \leqq \sup _{n \geqq 1} s^{p}\left(x_{n}\right) .
\end{aligned}
$$

Thus, by (7),

$$
s^{p}(x)=\lim _{m \rightarrow \infty} s_{m}^{p}(x) \leqq \sup _{n \geqq 1} s^{p}\left(x_{n}\right)<+\infty
$$


(10): We may assume $p>0$. If $d^{p}(x, y)=+\infty$ for every $y \neq x$, then by (1),

$$
s^{p}(x) \geqq d^{p}(x, w)-s^{p}(w)=+\infty .
$$

This proves one direction.

If $s^{p}(x)=+\infty$ and $d^{p}(x, y)<+\infty$ for some $y$, then assume $p$ is minimal. We have $x \in X$ and so we may choose a sequence $f_{n} \in R_{0}(X, 1)$ such that

$$
\left|f_{n}^{(p)}(x)\right| \longrightarrow+\infty,
$$

while $\left|f_{n}^{(p)}(x)-f_{n}^{(p)}(y)\right| \leqq M$ for all $n$, for some constant $M$. Form

$$
g_{n}(z)=(2 z-x-y) f_{n}(z) .
$$

Then

$$
g_{n}^{(p)}(z)=2 p f_{n}^{(p-1)}(z)+(2 z-x-y) f_{n}^{(p)}(z)
$$

Thus

$$
\begin{aligned}
\left|g_{n}^{(p)}(x)-g_{n}^{(p)}(y)\right| & \\
= & \left|2 p f_{n}^{(p-1)}(x)+(x-y) f_{n}^{(p)}(x)-2 p f_{n}^{(p-1)}(y)-(y-x) f_{n}^{(p)}(y)\right| \\
\geqq & |x-y|\left|f_{n}^{(p)}(x)+f_{n}^{(p)}(y)\right| \\
& -2 p\left|f_{n}^{(p-1)}(x)-f_{n}^{(p-1)}(y)\right| \longrightarrow+\infty \text { as } n \longrightarrow+\infty .
\end{aligned}
$$

(11): The point $x$ is an interior point of $x$ if and only if

$$
s_{n}(x) \leqq M^{n} n !
$$

for some constant $M>0$. ("Only if" is clear, and "if" is true because the inequality implies that every function in $R_{0}(X, 1)$ is actually analytic in a full disc centered at $x$. This forces $x \in X$.) (11) is just a way of rewriting this.

4. For our purposes all measures will be finite complex Borel regular measures with compact support in $C$. For $\nu>0$, the potential of order $\nu$ of a measure $\mu$ is given by

$$
\mu^{\nu}(z)=\int \frac{d|\mu|(\zeta)}{|\zeta-z|^{\nu}}
$$

where $|\mu|$ is the total variation measure of $\mu$. Wherever $\mu^{1}(z)<+\infty$ we define the Cauchy transform of $\mu$ by

$$
\widehat{\mu}(z)=\int \frac{d \mu(\zeta)}{\zeta-z} .
$$

For every continuous linear functional $L$ on $R(X)$ there is a measure 
$\mu$, supported on $X$, which "represents $L$ on $R(X)$ ", i.e.,

$$
\int f d \mu=L f
$$

for every $f \in R(X)$. This fact follows from the Hahn-Banach and Riesz Representation theorems. Also, $\mu$ may be chosen to have its support on $\partial X$, since $R(X)$ and $R(X) \mid \partial X$ are isomorphic Banach algebras. An annihilating measure for $R(X)$ is a measure $\mu$ on $X$ such that

$$
\int f d \mu=0
$$

for every $f \in R(X)$. We write $\mu \perp R(X)$. The following easy fact was first noted by Bishop, and plays a central role in our theory (cf. [2, p. 171]).

Lemma. If $\mu \perp R(X), \mu^{1}(y)<+\infty$, and $\hat{\mu}(y) \neq 0$, then the measure

$$
\frac{1}{\hat{\mu}(y)} \frac{1}{z-y} \mu
$$

represents "evaluation at $y$ " on $R(X)$, i.e.,

$$
\int f d \mu=f(y)
$$

for $f \in R(X)$.

The case $p=0$ of the following theorem is due to Browder [2, p. 176].

THEOREM 2. Let p be a nonnegative integer. Suppose the measure $\mu$ represents a bounded pth order point derivation on $R(X)$ at $x$. Then for every given $a>0$ there is a corresponding $b>0$ such that $d^{p}(x, y)<a$ whenever

$$
\sum_{\nu=1}^{p+1}|x-y|^{\nu} \mu^{\nu}(y)<b
$$

Proof. We proceed by induction on $p$ : Suppose $p$ is the least nonnegative integer for which the proposition fails. Let $\mu$ represent $D_{x}^{p}$ and $a>0$ be given. We may suppose $a<1$. For $\tau=0,1, \cdots$, $p-1, R(X)$ admits a bounded $\tau$ th order point derivation at $x$, represented by 


$$
\mu_{\tau}=\frac{\tau !(z-x)^{p-\tau}}{p !} \mu,
$$

so there are numbers $b_{\nu}>0$ such that

$$
\sum_{\nu=0}^{\tau+1}|x-y|^{\nu} \mu_{\tau}^{\nu}(y)<b_{\tau}
$$

forces $d^{\tau}(x, y)<a / 2$. Now

$$
\begin{aligned}
\mu_{\tau}^{\nu}(y) & =\int \frac{\tau !|z-x|^{p-\tau}}{|z-y|^{\nu}} d|\mu|(z) \\
& \leqq \tau !(\operatorname{diam} X)^{p-\tau} \mu^{\nu}(y),
\end{aligned}
$$

so, setting $c_{\tau}=b_{\tau}\left\{\sup _{0 \leqq \tau \leqq p} \tau \text { ! }(\operatorname{diam} X)^{p-\tau}\right\}^{-1}$, and $c=\inf _{0 \leqq \tau \leqq p-1} c_{\tau}$, we deduce that $\sum_{\nu=0}^{p+1}|x-y|^{\nu} \mu^{\nu}(y)<c$ forces (3) for $\tau=0,1, \cdots, p-1$.

Let $K=1+\exp (\operatorname{diam} X)$,

$$
T=2\left\{\sup _{0 \leqq \tau \leqq p} s^{\nu}(x)\right\} K .
$$

Note that $T \geqq 2 K$, since $s^{0}(x)=1$.

Choose $b>0$ to be smaller than each of the numbers $c, 1 / 2$, $p !(\operatorname{diam} X)^{-p-1}$ and $a\left\{2 T\left(K_{p}+\|\mu\|\right)\right\}^{-1}$, where $K_{p}>0$ is a constant, depending only on $p$, which will be described later.

Let (2) hold. We will show that $d^{p}(x, y)<a$. We claim it suffices to show

$$
d_{0}^{p}(y, x)<a / T .
$$

For, assuming (4), we have by Theorem 1(6), (1),

$$
\begin{aligned}
d^{p}(x, y) & \leqq K\left\{\sup _{0 \leqq \nu \leqq p} s^{\nu}(y)\right\} d_{0}^{p}(y, x) \\
& \leqq K\left\{\sup _{0 \leqq \nu \leqq p} s^{\nu}(x)+\sup _{0 \leqq \nu \leqq p} d^{\nu}(x, y)\right\} d_{0}^{p}(y, x) .
\end{aligned}
$$

Thus, if $d^{p}(x, y) \geqq a$, then $d^{p}(x, y)=\sup _{0 \leqq \nu \leqq p} d^{\nu}(x, y)$, since (3) holds for $\tau=0,1, \cdots, p-1$, so

$$
d^{p}(x, y)\left\{1-K d_{0}^{p}(y, x)\right\} \leqq \frac{1}{2} T d_{0}^{p}(y, x) .
$$

Since $K d_{0}^{p}(y, x)<a K / T<a / 2<1 / 2$, we deduce

$$
d^{p}(x, y) \leqq T d_{0}^{p}(y, x)<a,
$$

which is a contradiction.

We proceed to get (4).

The measure $\mu_{0}=\left((z-x)^{p} / p !\right) \mu$ represents evaluation at $x$ on $R(X)$. Thus $\sigma=(z-x) \mu_{0}$ annihilates $R(X)$. Now 


$$
\hat{\sigma}(y)=\frac{1}{p !} \int \frac{(z-x)^{p+1}}{z-y} d \mu(z)=1+(y-x) \hat{\mu}_{0}(y),
$$

so, since

$$
\begin{aligned}
\left|(y-x) \hat{\mu}_{0}(y)\right| & \leqq|y-x| \mu_{0}^{1}(y) \\
& \leqq \frac{|y-x|(\operatorname{diam} X)^{p+1} \mu^{1}(y)}{p !}<b<1,
\end{aligned}
$$

we have $\hat{\sigma}(y) \neq 0$. Also $\sigma^{1}(y)<+\infty$, since $\mu^{1}(y)<+\infty$, by (2). Thus, by the lemma, the measure

$$
\frac{\sigma}{\hat{\sigma}(y)(z-y)}=\frac{(z-x)^{p+1} \mu}{p ! \hat{\sigma}(y)(z-y)}
$$

represents evaluation at $y$ on $R(X)$, so

$$
\frac{(z-x)^{p+1} \mu}{\hat{\sigma}(y)(z-y)^{p+1}}
$$

annihilates the class

$$
B=\left\{f \in R_{0}(X, 1) \mid f(y)=f^{\prime}(y)=\cdots=f^{(p)}(y)=0\right\},
$$

since $\mu^{p+1}(y)<+\infty$, by (2).

Let $e=\hat{\sigma}(y)$. Then $|e|>1-b>1 / 2$, and also $|1-e|<b$.

We have

$$
\begin{aligned}
d_{0}^{p}(y, x) & =\sup \left\{\left|f^{(p)}(x)\right| f \in B\right\} \\
& =\sup \left\{\left|\int f(z)\left\{1-\frac{(z-x)^{p+1}}{e(z-y)^{p+1}}\right\} d \mu(z)\right| \mid f \in B\right\} \\
& \leqq \int\left|\frac{e(z-y)^{p+1}-(z-x)^{p+1}}{e(z-y)^{p+1}}\right| d|\mu|(z) \\
& \leqq \frac{1}{1-b} \int\left|\frac{(z-y)^{p+1}-(z-x)^{p+1}}{(z-y)^{p+1}}-(1-e)\right| d|\mu|(z) \\
& \leqq 2|x-y| \int\left|\sum_{\nu=0}^{p}\left(\begin{array}{l}
p \\
\nu
\end{array}\right) \frac{(z-x)^{\nu}}{(z-y)^{\nu+1}}\right| d|\mu|(z)+2 b\|\mu\| .
\end{aligned}
$$

Now we observe that $(z-x)^{\nu} /(z-y)^{\nu+1}$ is a linear combination of terms

$$
\frac{1}{z-y}, \frac{x-y}{(z-y)^{2}}, \cdots, \frac{(x-y)^{\nu}}{(z-y)^{\nu+1}}
$$

so that we may continue the inequality:

$$
\leqq 2 K_{p}|x-y| \sum_{\nu=1}^{p+1}|x-y|^{\nu-1} \mu^{\nu}(y)+2 b\|\mu\|,
$$


where $K_{p}$ depends only on $p$, and so, continuing:

$$
\begin{aligned}
& \leqq 2\left(K_{p}+\|\mu\|\right) b \\
& \leqq \frac{a}{T} .
\end{aligned}
$$

This concludes the proof.

5. We now establish a convergence theorem for the $d^{p}$ metric.

THeOREm 3. Suppose $p=0$, and $x$ is not a peak point for $R(X)$, or $p \geqq 1$, and $R(X)$ admits a bounded pth order point derivation at $x$. Suppose there is a positive constant $K$, and a sequence of points $\left\{y_{n}\right\}$, elements of $\dot{X}$, which converges to $x$ (in Euclidean norm), such that

$$
\operatorname{dist}\left[y_{n}, \partial X\right] \geqq K\left|y_{n}-x\right|
$$

for $n=1,2,3, \cdots$. Then $\left\{y_{n}\right\}$ converges to $x$ in the $d^{p}$ metric.

Proof. Select a measure $\mu$, supported on $\partial X$, with no mass at $x$, which represents the $p$ th order derivation at $x$.

By Theorem 2, it suffices to show that $\left|x-y_{n}\right|^{\nu} \mu^{\nu}\left(y_{n}\right)$ is small for each $\nu, 1 \leqq \nu \leqq p+1$, provided $n$ is large.

Fix $\varepsilon>0$, and $\nu, 1 \leqq \nu \leqq p+1$. If $z \in \partial X$, then for each $n \geqq 1$,

$$
\frac{\left|z-y_{n}\right|}{\left|x-y_{n}\right|} \geqq K
$$

by (3). Choose $r_{1}>0$ such that

$$
\mu B\left(x, r_{1}\right)<\frac{\varepsilon K}{2} .
$$

Choose $r>0$ such that

$$
\frac{r}{r_{1}}<\min \left\{\frac{\varepsilon}{2^{\nu+1}\|\mu\|}, \frac{1}{2}\right\} .
$$

Then choose $N$ so large that $n \geqq N$ ensures $\left|x-y_{n}\right|<r$. Then, for $n \geqq N$,

$$
\begin{aligned}
\left|x-y_{n}\right|^{\nu} \mu^{\nu}\left(y_{n}\right) & =\left|x-y_{n}\right|^{\nu} \int \frac{d|\mu|(z)}{\left|z-y_{n}\right|^{\nu}} \\
& =\left|x-y_{n}\right|^{\nu}\left\{\int_{C \backslash B\left(x, r_{1}\right)}+\int_{B\left(x, r_{1}\right)}\right\} \\
& \leqq \frac{r^{\nu} \| \mu||}{\left(r_{1} / 2\right)^{\nu}}+\frac{\mu B\left(x, r_{1}\right)}{K^{\nu}} \\
& \leqq \frac{\varepsilon}{2}+\frac{\varepsilon}{2}=\varepsilon .
\end{aligned}
$$


This completes the proof.

Corollary 1. Suppose $\dot{X}$ satisfies a cone condition at a point $x \in \partial X$. Then whenever $p=0$ and $x$ is not a peak point for $R(X)$, or $p \geqq 1$ and $R(X)$ admits a bounded pth order point derivation at $x$, it follows that $d^{p}(y, x) \rightarrow 0$ as $y$ approaches $x$ along the midline of the cone.

This clearly follows from Theorem 3. Using the language of tangent cones [3, p. 233] we can say more.

Corollary 2. Let $x \in \partial X, E$ be a compact connected subset of $X$, $x \in E, E \backslash\{x\} \subset \dot{X}$, and suppose that

$$
\operatorname{Tan}(E, x) \cap \operatorname{Tan}(\partial X, x)=(0) .
$$

Then under the same hypothesis on $p, R(X)$ as before, $d^{p}(y, x) \rightarrow 0$ as $y$ approaches $x$ in $E$.

Corollary 3. Suppose $\dot{X}$ satisfies a cone condition at $x$, and $\Gamma$ is the midline of the cone. Suppose $R(X)$ admits a bounded $p^{\text {th }}$ order point derivations at $x(p \geqq 1)$. Let $D_{x}^{p}$ and $D_{x}^{p-1}$ denote the normalized point derivations of orders $p$ and $p-1$ at $x$. Then

$$
D_{x}^{p} f=\lim _{\substack{y \rightarrow x \\ y \in \Gamma}}\left[\frac{f^{(p-1)}(y)-D_{x}^{p-1} f}{y-x}\right]
$$

for every $f \in R(X)$, and the convergence is equiconvergence on $R(X, 1)$.

This follows readily from Corollary 1.

6. For examples to which these results apply, see [5], [10]. Hallstrom [6] has given necessary and sufficient conditions that $R(X)$ admit a bounded point derivation at a point $x$. Essentially, the complement of $X$ has to be "thin" at $x$, in terms of analytic capacity.

Let $a_{n}, r_{n}$ be two sequences of positive numbers such that

$$
1>a_{n}+r_{n}>a_{n}>a_{n}-r_{n}>a_{n+1}+r_{n+1},
$$

for $n=1,2,3, \cdots$. Let $D_{n}$ denote the open disc with centre $a_{n}$ and radius $r_{n}$. Let $X$ be the compact set obtained by removing $\bigcup_{n=1}^{+\infty} D_{n}$ from the closed unit disc $D . \quad X$ is an example of a so-called $L$-set.

For these $L$-sets, the point 0 is a peak point for $R(X)$ if and only if $\sum_{n=1}^{+\infty} r_{n} / a_{n}=+\infty[10]$, and $R(X)$ admits a $p^{\text {th }}$ order bounded point derivation at 0 provided $\sum_{n=1}^{+\infty} r_{n} /\left(a_{n}^{p+1}\right)<+\infty$. Let $E$ denote the negative real axis. Applying Corollary 1 to $X$ we obtain the following: 
THEOREM 4.

(1) Suppose $\sum_{n=1}^{+\infty} r_{n} / a_{n}<+\infty$. Then $\lim _{\substack{z \rightarrow 0 \\ z \in E}}\left(d^{0}(z, 0)=0\right.$.

(2) Suppose $\sum_{n=1}^{+\infty} r_{n} /\left(a_{n}^{p+1}\right)<+\infty$. Then $\lim _{\substack{z \rightarrow 0 \\ y \in E}} d^{p}(z, 0)=0$.

By choosing, say, $a_{n}=1 /(n+1), r_{n}=1(n+1)$ !! we can ensure that the hypothesis of (2) is satisfied for every $p \leqq 0$, so that $f^{(p)} z$ is equiconvergent to $f^{(p)}(0)$ on $R_{0}(X, 1)$, for every $p$.

One might wonder whether some kind of Browder density theorem might work for $p>0$ : if $R(X)$ admits a $p$ th order bounded point derivation at $x$, are there always other bounded derivations at nearby points? The answer is no: in [9] an example is constructed in which $R(X)$ admits a first order bounded point derivation at just one point. Moreover, this example can be modified to produce an example with a bounded point derivation of every order at that certain point, and no other bounded point derivations of any order $\geqq 1$ anywhere else.

What goes wrong? The following observation may clarify things. If $\mu$ represents a first order bounded point derivation on $R(X)$ at $x$ and $\mu^{2}(y)<+\infty$, set

$$
\begin{aligned}
& C=\int \frac{(z-x)^{2}}{z-y} d \mu(z), \\
& D=\int \frac{(z-x)^{2}}{(z-y)^{2}} d \mu(z) .
\end{aligned}
$$

Then, provided $C \neq 0$ and $D \neq 0$, the measure

$$
V=\left\{\frac{1}{C} \frac{(z-x)^{2}}{(z-y)^{2}}-\frac{1}{C D} \frac{(z-x)^{2}}{z-y}\right\} \mu
$$

represents a first order bounded point derivation on $R(X)$ at $y$. So this gives a sufficient condition for the existence of other derivations: $\left\{y \mid \mu^{2}(y)<+\infty, C \neq 0, D \neq 0\right\} \neq \varnothing$. Unfortunately $\mu^{2}$ is the potential associated with harmonic functions in $R^{4}$, and the associated capacity, $C^{2}$, vanishes on planar sets. So it is entirely possible, even likely, that $\mu^{2}(y) \equiv+\infty$ on spt $\mu$. In fact, $\mu^{2}(y)<+\infty$ if and only if

$$
\sum_{n=1}^{+\infty} 4^{n}|\mu|\left(A_{n}(y)\right)<+\infty
$$

where $A_{n}(y)=\left\{z\left|1 / 2^{n+1} \leqq\right| z-y \mid \leqq 1 / 2^{n}\right\}, n=1,2,3, \cdots$. Thus, for instance, if

$$
\varlimsup_{r \rightarrow 0} \frac{|\mu|(B(y, r))}{r^{2}}>0
$$

(i.e., $|\mu|$ has positive area density at $y$ ), then $\mu^{2}(y)=+\infty$.

Returning to the problem posed in $\S 1$, we note that for $x \in \partial\left(X^{\circ}\right)$, 
without some condition on $\dot{X}$, we cannot ensure that there will be a sequence $x_{n} \rightarrow x$ with $x_{n} \in \dot{X}$ and $f^{\prime}\left(x_{n}\right)$ equiconvergent to $f^{\prime}(x)$ on $R_{0}(X, 1)$, even when $s^{1}(x)<+\infty$. For let $X$ be the example of [9], with a bounded point derivation just at 0 , and select any sequence $\left\{x_{n}\right\}$ of distinct points of $X$, tending to 0 . For each $n(n=1,2,3, \cdots)$ there is a function $f_{n} \in R_{0}(X, 1)$ such that $f_{n}^{\prime}\left(x_{n}\right)>4 n$. Inductively, choose a closed disc $D_{n}$ centered at $x_{n}$ such that $f_{n}$ is analytic in a neighborhood of $D_{n},\left\|f_{n}\right\|_{D_{n}} \leqq 2,\left|f_{n}^{\prime}(z)\right|>2 n$ for $z \in D_{n}, D_{n} \cap D_{m}=\varnothing$ for $m<n, x_{m} \notin D_{n}$ for $m>n$. Form a new compact set $Y=X \cup$ $\left(\mathrm{U}_{n=1}^{+\infty} D_{n}\right)$. Then $R(Y)$ still admits a bounded point derivation at 0 . The only other bounded point derivations are at points of the $D_{n}$. For $z \in D_{n}, s^{1}(z)>n$. So there is no sequence of points of $\dot{Y}=\bigcup_{n=1}^{+\infty} \dot{D}_{n}$ along which $f^{\prime}$ is equiconvergent to $f^{\prime}(0)$ on $R_{0}(Y, 1)$.

7. Let $X$ be a compact subset of the plane. Let $A$ be an algebra of functions on $C$ which contains the polynomial and all of whose functions are analytic on $\dot{X}$. Suppose $A$, regarded as a subset of $C(X)$, forms a function algebra. Suppose $A$ enjoys the Arens property: For each $x \in X$,

$$
A_{x}=\{f \in A \mid f \text { is analytic on a neighborhood of } x\}
$$

is dense in $A$ in the uniform norm on $X$. (A sufficient condition for this is that $A$ contains a dense subset $B$ which is " $T_{\phi}$-invariant", i.e., the function $T_{\phi} f$, given by

$$
T_{\phi} f(z)=\frac{1}{\pi} \int \frac{f(z)-f(\zeta)}{z-\zeta} \frac{\partial \phi(\zeta)}{\partial \zeta} d \mathscr{L}^{2}(\zeta)
$$

belongs to $B$ whenever $f$ belongs to $B$ and $\phi$ is a continuously differentiable function with compact support. An example is $A=A(X)$, the algebra of all continuous functions on $C$ which are analytic on $X$; another example is $A=A^{\alpha}(X)$, the uniform closure on $X$ of those functions in $A(X)$ which satisfy a condition Lip $\alpha$ on $C$.) Then most of what we have done for $R(X)$ goes through for $A$. New functions $d^{p}, s^{p}, d_{0}^{p}$ may be defined analogously, for instance:

$$
\begin{aligned}
d^{p}(x, y)= & \sup \left\{\mid f^{(p)}(x)-f^{(p)}(y)\|f \in A,\| f \|_{x} \leqq 1,\right. \\
& f \text { is analytic on a neighborhood of }\{x, y\}\} .
\end{aligned}
$$

For any $x \in C$ we can form $A_{x}$. So given any compact set $Y \subset C$ we may form a new algebra

$$
\left.Y(A)=\bigcap_{x \in Y} \text { (Uniform closure on } Y \text { of } A_{x} \cap A(Y)\right) .
$$

$Y(A)$ is clearly a uniform algebra on $Y$, contains the polynomials, and all its functions are analytic on $\dot{Y}^{\circ}$. Moreover, by its definition, 
it has the Arens property.

Replacing $R(X)$ by $A$, Theorem 1 will go through, except that (7) will have to be changed:

( $\left.7^{\prime}\right)$ if $x \in \partial X, V_{n}$ is a decreasing sequence of compact neighborhoods of $x$, whose intersection is $\{x\}$, and $X_{n}=X \cup V_{n}$, then $s_{n}^{p}(x) \uparrow s^{p}(x)$, and $d_{n}^{p}(x, \cdot) \uparrow d^{p}(x, \cdot)$, where $s_{n}^{p}$ and $d_{n}^{p}$ are the $s^{p}$ and $d^{p}$ functions associated with the algebras $X_{n}(A)$.

Lemma 1 goes through, using the Arens property.

The maximal ideal space of $A$ is $X$ (cf. [1], its Silov boundary is a subset of $\partial X$, so Theorems 2 and 3 work for $A$ in place of $R(X)$.

8. Now we turn to $H^{\circ}(U)$, the Banach algebra of bounded analytic functions (with $L^{\infty}$ norm) on the bounded open set $U \subset C$. First, we look at $H^{\infty}(U)$ itself. There is a natural projection map from the maximal ideal space $\mathscr{C}$ of $H^{\infty}(U)$ to $\bar{U}$, given by $\phi \rightarrow \phi(z)$ (recall that $z$ denotes the identity map of $C$ ). The fiber $\mathscr{C}_{x}$ over a point $x \in U$ consists of one point $\phi_{x}=$ evaluation at $x$. The fiber $\mathscr{C}_{x}$ over a point $x \in \partial U$ is usually very large. Gamelin and Garnett [5] showed that a necessary and sufficient condition for $\mathscr{A}_{x}$ to be a peak set for $H^{\infty}(U)$ is that

$$
\sum_{n=1}^{+\infty} 2^{n} \gamma\left(A_{n}(x) \backslash U\right)=+\infty .
$$

Here $\gamma$ denotes the analytic capacity:

$$
\gamma(K)=\sup \left\{\left|f^{\prime}(\infty)\right| \mid f \text { is analytic off } K,\|f\| \leqq 1, f(\infty)=0\right\} .
$$

When $\mathscr{A}_{x}$ is not a peak set, they showed that it contains a distinguished homomorphism, $\phi_{x}$, characterized by the property that it has a representing measure on $\mathscr{M}$ with no mass on $\mathscr{K}_{x}$.

We say that an element $D \in H^{\infty}(U)^{*}$, a continuous linear map of $H^{\infty}(U)$ to $C$, is a first order bounded point derivation at a point $\phi \in \mathscr{M}$ if

$$
D(f g)=\phi(f) D g+\phi(g) D f
$$

whenever $f, g \in H^{\infty}(U)$. $\quad D$ is called regular if $D z \neq 0$, and a regular $D$ is normalised if $D z=1$. We shall be concerned with regular derivations only, but we note that there are usually many derivations on $H^{\infty}(U)$ which annihilate $z$. For instance, let $U$ be the open unit disc. Then Hoffman [7] has shown that the fiber $\mathscr{C}_{1}$ over the point $1 \in \partial U$ contains many homeomorphic images of the unit disc, on each 
of which all the functions in $H^{\infty}(U)$ are analytic. So there is a superabundance of bounded point derivations at points of $\mathscr{M}_{1}$, and each of these derivations annihilates $z$.

Inductively, we say $H^{\infty}(U)$ admits a regular normalized $p$ th order bounded point derivation at $\phi \in \mathscr{M}$ if the following hold:

(1) For each $\nu, 1 \leqq \nu \leqq p-1, D^{\nu}$ is a $\nu$ th order regular normalized bounded point derivation at $\phi$.

(2) There is an element $D^{p} \in H^{\infty}(U)^{*}$ such that

$$
D^{p}(f g)=\sum_{\nu=0}^{p}\left(\begin{array}{c}
p \\
\nu
\end{array}\right) D^{\nu} f D^{p-\nu} g
$$

for all $f, g \in H^{\infty}(U)$, where $D^{0} f$ means $\phi(f)$.

(3) $D^{p} z^{p}=p$ !

We observe that for $p \geqq 1$ there cannot be any regular $p$ th order bounded point derivation at a point $\phi \in \mathscr{C}_{x} \backslash\left\{\phi_{x}\right\}$. For such a derivation would have a representing measure $\mu$ on $\mathscr{L}$, and then $\left((z-x)^{p} / p !\right) \mu$ would be a representing measure for $\phi$ with no mass on $\mathscr{M}_{x}$, which is impossible.

Theorem 5. Let $x \in U, p \geqq 1$. Then $H^{\infty}(U)$ admits a regular bounded pth order point derivation at the distinguished homomorphism $\phi_{x}$ in the fiber over $x$ if and only if

$$
\sum_{n=1}^{+\infty} 2^{(p+1) n} \gamma\left(A_{n}(x) \backslash U\right)<+\infty .
$$

Proof. If (5) holds, then certainly (4) fails, so $\mathscr{A}_{x}$ is not a peak fiber and $\phi_{x}$ exists. By a device in Gamelin and Garnett's proof of the peak set criterion [5, p. 459, third paragraph], $U$ can be shrunk a little to produce a compact set $X$ with the properties:

(1) $X \subset U \cup\{x\}$,

(2) $x \in X$,

(3) $\sum_{n=1}^{+\infty} 2^{(p+1) n} r\left(A_{n}(x) \backslash X\right)<+\infty$.

By Hallstrom's Theorem [6, p. 156], $R(X)$ admits a (normalized) bounded point derivation of order $p$ at $x$. Choose a representing measure $\mu$ for this derivation with support on $X$ and no mass at $x$. Then, for $\nu=0,1, \cdots, p$ the measure $\mu_{\nu}=\left(\nu !(z-x)^{p-\nu} / p !\right) \mu$ represents a (normalized) $\nu$ th order bounded point derivation on $R(X)$ at $x$, if $\nu \geqq 1$, and $\mu_{0}$ represents $x$ and has no mass at $x$. Now any function in $H^{\infty}(U)$ which extends analytically to a neighborhood of $x$ belongs to $R(X)$, so for any two such functions, $f$ and $g$, we have

$$
\int f g d \mu=\sum_{\nu=0}^{p}\left(\begin{array}{l}
p \\
\nu
\end{array}\right) \int f d \mu_{\nu} \int g d \mu_{p-\nu} .
$$


Since, as is well-known [5, Cor. 2.2], the set of all such functions is pointwise boundedly dense in $H^{\infty}(U)$, the dominated convergence theorem implies that (6) holds for any $f, g \in H^{\infty}(U)$. Thus $\mu$ represents a regular bounded $p$ th order point derivation on $H^{\infty}(U)$ at $\phi_{x}$.

For the other direction, assume (5) fails. If $\mathscr{C}_{x}$ is a peak set there is no distinguished homomorphism, and nothing to prove. Otherwise, (4) fails, and we may, just as in Hallstrom's proof of his Theorem 1' [6, pp. 163-164], construct a sequence of functions $g_{n}$, each one in $H^{\infty}(U)$ and analytic in a neighborhood of $x$ such that $\left|g_{n}^{(p)}(x)\right|>n\|g\|_{\infty}$. Thus $H^{\infty}(U)$ cannot admit a $p^{\text {th }}$ order bounded point derivation at $\phi_{x}$. This proves the theorem.

We remark that there is at most one regular normalised bounded $p$ th order point derivation at a distinguished homomorphism $\phi_{x}$. For, from the proof of Theorem 5, any two agree on a dense subset of $H^{\infty}(U)$, and have representing measures with no mass on $\mathscr{C}_{x}$. Thus, by dominated convergence, they coincide.

9. The zero order Gleason metric $d^{0}$ on the maximal ideal space of $H^{\infty}(U)$ is given by

$$
d^{0}(\phi, \psi)=\sup \left\{|\phi(f)-\psi(f)| \mid f \in H^{\infty}(U),\|f\|_{U} \leqq 1\right\} .
$$

To define the higher order metrics, we take first the case where $\phi$ and $\psi$ are distinguished homomorphisms at each of which $H^{\infty}(U)$ admits normalised regular bounded $p^{\text {th }}$ order point derivations $D_{\phi}^{p}$ and $D_{\varphi}^{p}$. Then

$$
d^{p}(\phi, \psi)=\sup \left\{\left|D_{\phi}^{p} f-D_{\psi}^{p} f\right| \mid f \in H^{\infty}(U),\|f\|_{U} \leqq 1\right\} .
$$

In all other cases, we set $d^{p}(\phi, \psi)=+\infty$. Let $s^{p}(\phi)$ be the norm of $D_{\phi}^{p}$, if this exists, otherwise $s^{p}(\phi)=+\infty$. For points $y \in U$ we will write $y$ for "evaluation at $y$ ".

THEOREM 6. Let $p \geqq 1$. Suppose there is a constant $K>0$ and a sequence of points $y_{n} \in U,\left|y_{n}-x\right| \rightarrow 0$ as $n \rightarrow+\infty$, such that

$$
\operatorname{dist}\left[y_{n}, \partial U\right] \geqq K\left|y_{n}-x\right| \text {. }
$$

Suppose $H^{\infty}(U)$ admits a regular $p^{\text {th }}$ order bounded point derivation at the distinguished homomorphism $\phi_{x}$ over $x$. Then $d^{p}\left(y_{n}, \dot{\phi}_{x}\right) \rightarrow 0$ as $n \rightarrow+\infty$.

Proof. We shall deduce this from Theorem 3. As in Theorem 5, we may shrink $U$ to a compact set $X$ which satisfies the hypotheses of Theorem 3, with a smaller $K$. Thus there are representing meas- 
ures $\mu_{n}$ for the $D_{y_{n}}^{p}$, and $\mu$ for $D_{\phi_{x}}^{p}$, with closed support in $U \cup \mathscr{C}_{x}$ and no mass on $\mathscr{M}_{x}$ such that

$$
\int f d \mu_{n} \longrightarrow \int f d \mu
$$

uniformly for $f \in R(X)$. Again, since $R_{0}(X)$ is pointwise boundedly dense in $H^{\infty}(U)$, this means that $D_{y_{n}}^{p} f=\int f d \mu_{n}$ is equiconvergent to $D_{\varphi_{x}}^{p} f=\int f d \mu$ for all $f \in H^{\infty}(U)$.

The analogous result when $p=0$ (also a corollary of Theorem 3) is due to Gamelin and Garnett [5, 5.1].

\section{REFERENCES}

1. R. Arens, The maximal ideals of certain function algebras, Pacific J. Math., 8 (1958), 641-648.

2. A. Browder, Introduction to Function Algebras, Benjamin, 1969.

3. H. Federer, Geometric Measure Theory, Springer, 1969.

4. T. Gamelin, Uniform Algebras, Prentice-Hall, 1969.

5. T. Gamelin and J. Garnett, Distinguished homomorphisms and fiber algebras, Amer. J. Math., 92 (1970), 455-474.

6. A. Hallstrom, On bounded point derivations and analytic capacity, J. Functional Analysis, 4 (1969), 153-165.

7. Kenneth Hoffman, Banach Spaces of Analytic Functions, Prentice-Hall, 1962.

8. M. S. Melnikov, Structure of the Gleason parts of the algebra $R(E)$, Functional Ana. Appl., 1 (1967), 84-86.

9. A. O'Farrell, An isolated bounded point derivation, Proceedings Amer. Math. Soc., 39 (1973), 559-562.

10. L. Zalcman, Analytic Capacity and Rational Approximation, Springer-Verlag Lecture Notes 50, 1968.

Received April 12, 1973. This research was supported in part by the National Science Foundation under grant GP-28574(X01).

BRown University 


\section{PACIFIC JOURNAL OF MATHEMATICS}

\section{EDITORS}

RICHARD ARENS (Managing Editor)

University of California

Los Angeles, California 90024

\section{J. DUGUNDJI}

Department of Mathematics University of Southern California Los Angeles, California 90007

D. Gilbarg and J. Milgram

Stanford University

Stanford, California 94305

University of Washington
Seattle, Washington 98105

ASSOCIATE EDITORS
E. F, BECKENBACH
B. H. NEUMANN
F. WOLF
K. Yoshida

\section{SUPPORTING INSTITUTIONS}

\author{
UNIVERSITY OF BRITISH COLUMBIA \\ CALIFORNIA INSTITUTE OF TECHNOLOGY \\ UNIVERSITY OF CALIFORNIA \\ MONTANA STATE UNIVERSITY \\ UNIVERSITY OF NEVADA \\ NEW MEXICO STATE UNIVERSITY \\ OREGON STATE UNIVERSITY \\ UNIVERSITY OF OREGON \\ OSAKA UNIVERSITY
}

\author{
UNIVERSITY OF SOUTHERN CALIFORNIA \\ STANFORD UNIVERSITY \\ UNIVERSITY OF TOKYO \\ UNIVERSITY OF UTAH \\ WASHINGTON STATE UNIVERSITY \\ UNIVERSITY OF WASHINGTON \\ * * * * \\ AMERICAN MATHEMATICAL SOCIETY \\ NAVAL WEAPONS CENTER
}

The Supporting Institutions listed above contribute to the cost of publication of this Journal, but they are not owners or publishers and have no responsibility for its content or policies.

Mathematical papers intended for publication in the Pacific Journal of Mathematics should be in typed form or offset-reproduced, (not dittoed), double spaced with large margins. Underline Greek letters in red, German in green, and script in blue. The first paragraph or two must be capable of being used separately as a synopsis of the entire paper. Items of the bibliography should not be cited there unless absolutely necessary, in which case they must be identified by author and Journal, rather than by item number. Manuscripts, in duplicate if possible, may be sent to any one of the four editors. Please classify according to the scheme of Math. Rev. Index to Vol. 39. All other communications to the editors should be addressed to the managing editor, or Elaine Barth, University of California, Los Angeles, California, 90024.

100 reprints are provided free for each article, only if page charges have been substantially paid. Additional copies may be obtained at cost in multiples of 50 .

The Pacific of Journal Mathematics is issued monthly as of January 1966. Regular subscription rate: $\$ 72.00$ a year (6 Vols., 12 issues). Special rate: $\$ 36.00$ a year to individual members of supporting institutions.

Subscriptions, orders for back numbers, and changes of address should be sent to Pacific Journal of Mathematics, 103 Highland Boulevard, Berkeley, California, 94708.

PUBLISHED BY PACIFIC JOURNAL OF MATHEMATICS, A NON-PROFIT CORPORATION

Printed at Kokusai Bunken Insatsusha (International Academic Printing Co., Ltd.), 270, 3-chome Totsuka-cho, Shinjuku-ku, Tokyo 160, Japan.

Copyright (C) 1973 by Pacific Journal of Mathematics Manufactured and first issued in Japan 


\section{Pacific Journal of Mathematics}

\section{Vol. 53, No. $2 \quad$ April, 1974}

Kenneth Abernethy, On characterizing certain classses of first countable spaces by

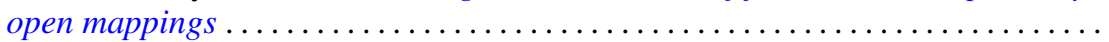

Ross A. Beaumont and Donald Lawver, Strongly semisimple abelian groups .......

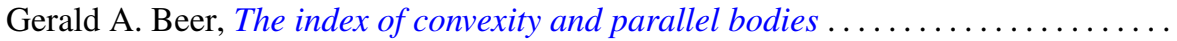

Victor P. Camillo and Kent Ralph Fuller, On Loewy length of rings ..............

Stephen LaVern Campbell, Linear operators for which $T^{*} T$ and $T T^{*}$ commute.

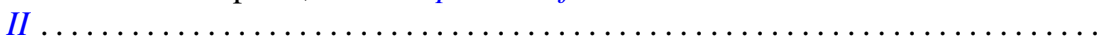

Charles Kam-Tai Chui and Philip Wesley Smith, Characterization of a function by

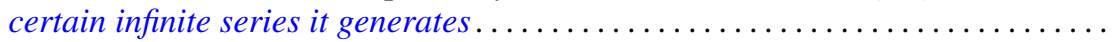

Allan L. Edelson, Conjugations on stably almost complex manifolds . ...........

Patrick John Fleury, Hollow modules and local endomorphism rings . . ..........

Jack Tilden Goodykoontz, Jr., Connectedness im kleinen and local connectedness in

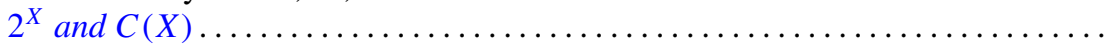

Robert Edward Jamison, II, Functional representation of algebraic intervals .......

Athanassios G. Kartsatos, Nonzero solutions to boundary value problems for

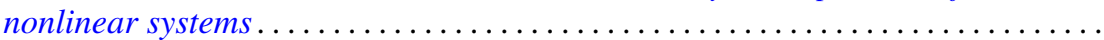

Soon-Kyu Kim, Dennis McGavran and Jingyal Pak, Torus group actions on simply

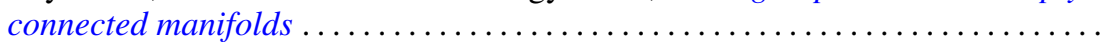

David Anthony Klarner and R. Rado, Arithmetic properties of certain recursively

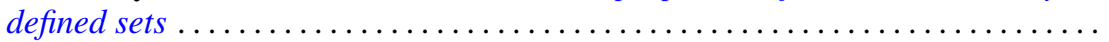

Ray Alden Kunze, On the Frobenius reciprocity theorem for square-integrable

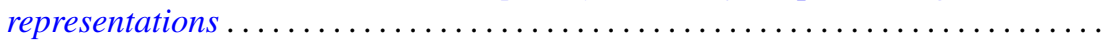

John Lagnese, Existence, uniqueness and limiting behavior of solutions of a class of differential equations in Banach space...

Teck Cheong Lim, A fixed point theorem for families on nonexpansive mappings Lewis Lum, A quasi order characterization of smooth continua

Andy R. Magid, Principal homogeneous spaces and Galois extensions . .

Charles Alan McCarthy, The norm of a certain derivation ..... . .

Louise Elizabeth Moser, On the impossibility of obtaining $S^{2} \times S^{1}$ by elementary surgery along a knot. .

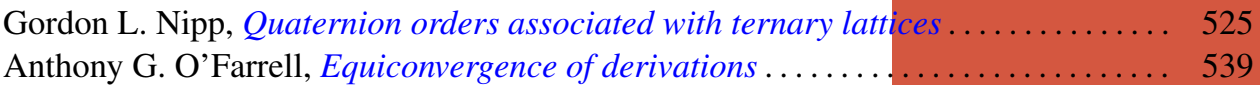

Dorte Olesen, Derivations of $A W^{*}$-algebras are inner . . . . . . . . . . . . . . . 555

Dorte Olesen and Gert Kjærgaard Pedersen, Derivations of $C^{*}$-algebras have

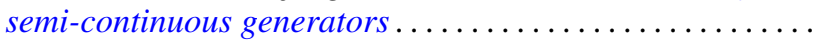

Duane O’Neill, On conjugation cobordism.

Chull Park and S. R. Paranjape, Probabilities of Wiener paths crossing differentiable

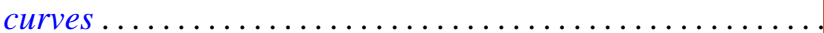

Edward Ralph Rozema, Almost Chebyshev subspaces of $L^{1}(\mu$;

Lesley Millman Sibner and Robert Jules Sibner, A note on the Atiyah-Bott fixed

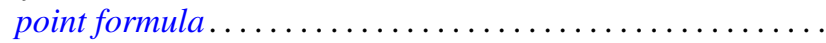

Betty Salzberg Stark, Irreducible subgroups of orthogonal groups generated by

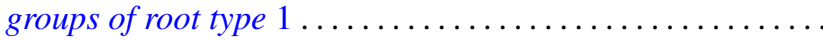

N. Stavrakas, A note on starshaped sets, $(k)$-extreme points and the half ray

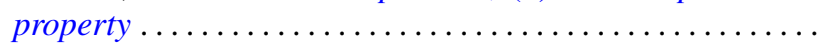

Carl E. Swenson, Direct sum subset decompositions of $Z \ldots \ldots$ 